\title{
TOTAL OSSICULAR REPLACEMENT PROSTHESIS OF THE MIDDLE EAR: A BIOMECHANICAL ANALYSIS
}

\author{
FERNANDA GENTIL* \\ IDMEC, Clinica ORL-Dr. Eurico Almeida \\ Widex, ESTSP, Rua Dr. Roberto Frias 404 \\ 4200-465 Porto, Portugal \\ fernanda.fgnanda@gmail.com \\ MARCO MARQUES ${ }^{\dagger}$, MARCO PARENTE $^{\star}$, PEDRO MARTINS§, \\ CARLA SANTOS ${ }^{\uparrow}$ and RENATO NATAL JORGE \\ IDMEC, Faculdade de Engenharia da Universidade do Porto \\ Campus da FEUP: Rua Dr. Roberto Frias, 404, 4200-465 Porto, Portugal \\ †marco.costa.marques@gmail.com \\ ${ }^{\dagger}$ mparente@fe.up.pt \\ §palsm@fe.up.pt \\ Ifsantos.carla@gmail.com \\ Irnatal@fe.up.pt
}

The main goal of the present study is to analyze and characterize the behavior of the middle ear, when a total ossicular replacement prosthesis (TORP) is used in the ossicular chain, in order to troubleshoot conductive hearing loss. Using a finite element model (FEM), a dynamic study of the middle ear was made. The displacement values were obtained at the umbo and stapes footplate, for a sound pressure level of $80 \mathrm{~dB}$ sound pressure level (SPL) applied at the tympanic membrane, when a cartilage in membrane-prosthesis interface of different diameters and thicknesses was used. The results were compared with the healthy middle ear model. The usage of this model aims to achieve a set of techniques that promotes the best possible performance of prosthesis in the middle ear. The present study allows to conclude that the rehabilitation of the middle ear with TORP can lead to the best results when used with $4 \mathrm{~mm}$ diameter cartilages, with a thin thickness of $0.3 \mathrm{~mm}$.

Keywords: Biomechanics; finite element method; middle ear; TORP. 


\section{Introduction}

The major function of the auditory system is to convert pressure variations, created by sound wave propagation, into bioelectrical signals. Complications in the normal functioning of the human ear are, an obstacle to a normal conversion process thus creating a deafness problem. This deafness problem is related to hearing loss, which is a full or partial decrease in the ability to detect or understand sounds. Hearing impairment is a condition that can occur at any period of life, to any gender and to any race, with many origins, having a significant repercussions in human lifestyle. ${ }^{1}$ It can be defined according to its severity, lesion foci, uni or bilaterality and to the frequencies that are lost. ${ }^{2}$ Conductive hearing loss occurs when there is a pathology in the outer or/and in the middle ear. Those pathologies can be identified as the presence of earwax, malformations in the ossicular chain, otosclerosis, tumors, otitis or tympanic membrane perforation, among other. ${ }^{2}$

In conductive hearing loss, when there is an ossicular chain malformation, the Austin-Kartush classification defines the ossicular impairment in seven classes and the corresponding ossicular chain reconstruction technique. ${ }^{3}$

The prosthesis type used in the rehabilitation of the middle ear, total ossicular replacement prosthesis (TORP) or a partial ossicular replacement prosthesis (PORP) depends on the level of the ossicular impairment. ${ }^{3}$

In case of a TORP, the cartilage must be interposed between the tympanic membrane and the prosthesis. ${ }^{4-7}$ This cartilage can be extracted from tragus. ${ }^{6,7}$ The placement of the cartilage intends to reduce the extrusion rate of the TORP through the tympanic membrane. The fixation of this cartilage can be done by using sutures, "glued", placed on an available Polyethylene glycol (PEG), or simply overlaid as a dome. ${ }^{7}$ According to various authors, it can be held in place by water adhesion forces, but this methodology still leads to unsatisfying hearing, caused by unwanted displacements of the prosthesis. ${ }^{8,9}$ These methodologies can be characterized by the usage of centered holed cartilages fixed in the stapes footplate, and even pieces that make the connection of the prosthesis with the stapes. ${ }^{8}$

The first study about the biomechanical behavior of the middle ear, using the finite element model (FEM), was made in a cat, in $1978 .{ }^{10}$ Using FEM, the present study evaluates the sound transmission in the middle ear, when a TORP type prosthesis replaces the ossicular chain in order to solve problems of conductive hearing loss in a class D procedure of the Austin-Kartush classification. This class is characterized by the absence of the ossicular chain, except the stapes footplate. ${ }^{3}$ Thus, in order to understand the behavior of the middle ear when it is replaced by a TORP, a dynamic study was performed, using the ABAQUS software.

\section{Finite Element Analysis}

\subsection{FEM}

The FEM of the eardrum and ossicles (malleus, incus and stapes), ligaments and muscles, of the normal ear used, was built by Gentil et al. ${ }^{11,12}$ based on images of computerized tomography of a 65 -year-old woman. This initial model was used as the template for the model of this study, where the ossicular chain and respective muscles and ligaments are not present, and components like the cartilage and the 

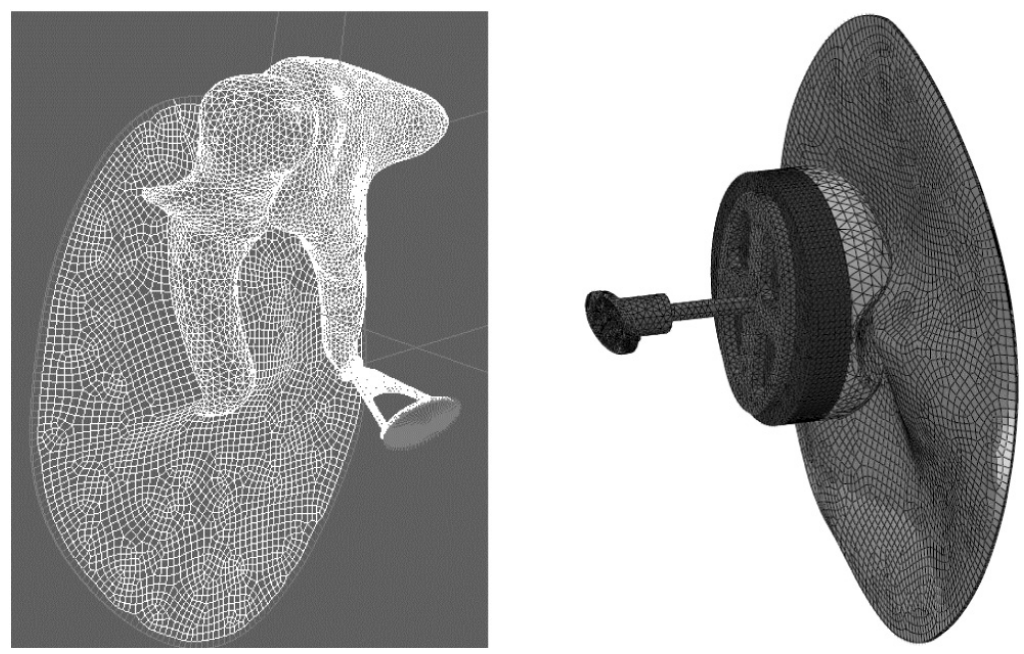

Fig. 1. Comparison between models of normal middle ear and the TORP.

TORP were added. This model was created in order to be within the specification of surgical techniques used and described by many authors. ${ }^{4-9}$ The Fig. 1 shows the comparison between the model representative of the normal middle ear and the model with TORP. The default ABAQUS direct solver for sparse matrices was used to solve the system of equations resulting from the FEM.

The cartilage used to interface between the TORP and the tympanic membrane has a cylindrical shape. The dimensions of the cartilage used was $0.3 \mathrm{~mm}$ and $0.7 \mathrm{~mm}$ thickness and of $4 \mathrm{~mm}$ and $5 \mathrm{~mm}$ in diameter. The elements of cartilage for all models are of type C3D4, in the ABAQUS software.

FEM were created for different lengths of TORP. TORP foot and head have always the same dimensions, but TORP rod must have different sizes due to different cartilage thicknesses.

The angle between prosthesis and stapes was considered of $10^{\circ}$.

The material properties for the middle ear model were used according to Gentil et al. ${ }^{12-14}$ In general the mechanical behavior of the model is elastic. For the ligaments and the muscles, the model has a nonlinear hyperelastic behavior, using the Yeoh model. ${ }^{15,16}$

The mechanical properties of the tragal cartilage were obtained in the literature according to Wen et al. ${ }^{17}$ The material of the TORP is characterized as being titanium grade 2 . The glue properties were obtained in the literature according to Schimidt et al. ${ }^{18}$ For the prosthesis, the Poisson ratio is 0.37 and for the glue is 0.4. The contacts between the geometrical models were of type-TIE and hard contact. The hard contact is built according to Newton-Raphson technique. The boundary conditions of the external nodes of the pars tensa ligaments, around the tympanic membrane, were fixed. This fixation has the objective to simulate the tympanic sulcus. 


\section{Results and Conclusions}

The results gathered from these models are relative to the displacements in two nodes, one node from the tympanic membrane and other from the stapes footplate. For a sound pressure level of $80 \mathrm{~dB}$ SPL (sound pressure of $0.2 \mathrm{~Pa}$ ) applied to the tympanic membrane, the results of the models with TORP were compared with a model of the healthy middle ear.

The results were obtained for the umbo and stapes footplate displacements, considering the diameters of $4 \mathrm{~mm}$ and $5 \mathrm{~mm}$ for the cartilage and also the values of $0.3 \mathrm{~mm}$ and $0.7 \mathrm{~mm}$ for the thickness.

The results of the umbo displacements are shown in Fig. 2, comparing the model representative of the normal middle ear with four models, where the ossicular chain was replaced by a TORP: Two cartilage disks with $4 \mathrm{~mm}$ diameter and $0.3 \mathrm{~mm}$ and $0.7 \mathrm{~mm}$ thickness; two cartilage disks with $5 \mathrm{~mm}$ diameter and $0.3 \mathrm{~mm}$ and $0.7 \mathrm{~mm}$ thickness.

In Fig. 3, one can see the stapes footplate displacements, comparing the normal model of the middle ear with the models where the ossicular chain was replaced by a TORP of $4 \mathrm{~mm}$ and $5 \mathrm{~mm}$ of diameter with thicknesses of $0.3 \mathrm{~mm}$ and $0.7 \mathrm{~mm}$.

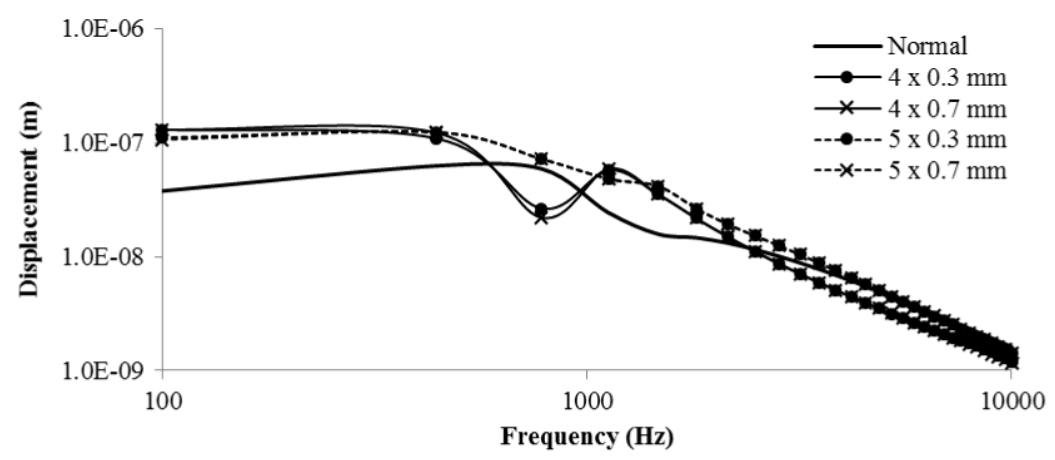

Fig. 2. Umbo displacements.

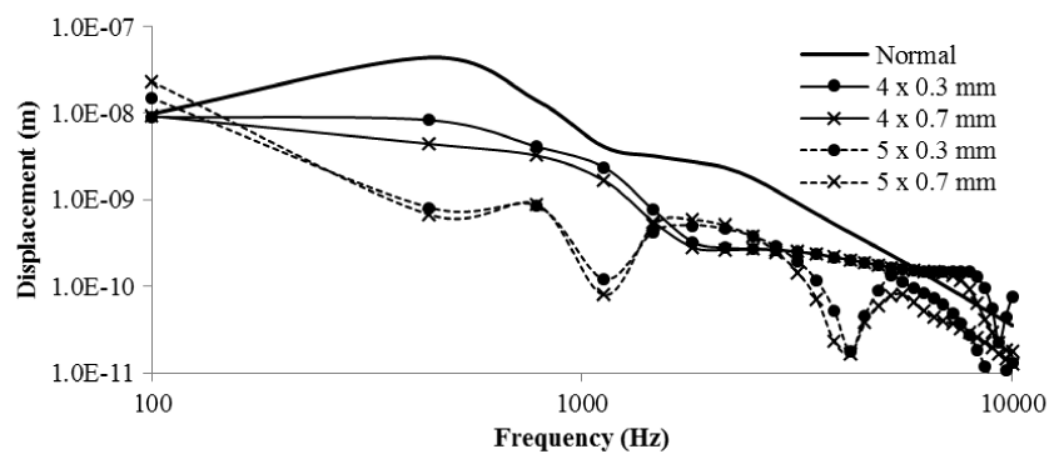

Fig. 3. Stapes footplate displacements. 
The most significant conclusion of this work is that a TORP-tympanic membrane cartilage interface with $4 \mathrm{~mm}$ diameter and a thickness of $0.3 \mathrm{~mm}$ allowed results closer to the reference results for the normal middle ear.

\section{Acknowledgments}

The authors acknowledge the funding of the project PEst-OE/EME/LA0022/2013, from Fundação da Ciência e Tecnologia, Portugal and to the project "Biomechanics: contributions to the healthcare", reference NORTE-07-0124-FEDER-000035 cofinanced by Programa Operacional Regional do Norte (ON.2-O Novo Norte), through the Fundo Europeu de Desenvolvimento Regional (FEDER). 


\section{References}

1. Gentil F, Estudo Biomecânico do Ouvido Médio, Faculdade de Engenharia da Universidade do Porto, Porto, 2008.

2. Canalis RF, Abemayor E, Shulman J, Blunt and penetrating injuries to the ear and temporal bone, in Canalis RF, Lambert PR (eds.), The Ear: Comprehensive Otology, Lippincott Williams \& Wilkins, Philadelphia, pp. 785-800, 2000.

3. Wiet RJ, Wiet RM, Experience-driven ossiculoplasty, operative techniques, Otolaryng Head Neck 21:211-216, 2010.

4. Cavaliere M, Mottola G, Rondinelli M, Iemma M, Tragal cartilage in tympanoplasty: Anatomic and functional results in 306 cases, Acta Oto-Laryngol 29:27, 2009.

5. Dornhoffer J, Cartilage tympanoplasty: Indications, techniques, and outcomes in a 1000 patient series, Laryngoscope 113:1844-1856, 2003.

6. Neff BA, Rizer FM, Schuring AG, Lippy WH, Tympano-ossiculoplasty utilizing the spiggle and theis titanium total ossicular replacement prosthesis, Laryngoscope 113:1525-1529, 2003.

7. Pyle GM, Ossicular sculpting for conductive hearing loss, operative techniques, Otolaryng Head Neck 14:237-242, 2003.

8. Beutner D, Hüttenbrink KB, Passive and active middle ear implants, GMS Curr Top Otorhinolaryng Head Neck 8: Doc.09, 2009.Published online 2011 Mar 10.

9. Beutner D, Luers JC, Huttenbrink KB, Cartilage 'shoe': A new technique for stabilisation of titanium total ossicular replacement prosthesis at centre of stapes footplate, $J$ Laryngol Otol 122:682-686, 2008.

10. Funnell WR, Laszlo CA, Modeling of the cat eardrum as a thin shell using the finiteelement method, J Acoust Soc Am 63:1461-1467, 1978.

11. Gentil F, Natal R, Parente M, Martins P, Ferreira A, Estudo biomecânico do ouvido médio, Clínica e Investigação em Otorrinolaringologia 3:24-30, 2009.

12. Gentil F, Parente M, Martins P, Garbe C, Jorge RN, Ferreira A, Tavares JMRS, The influence of mechanical behaviour of the middle ear ligaments: A finite element analysis, J Eng Med 225:68-76, 2011.

13. Gentil F, Garbe C, Parente M, Martins P, Ferreira A, Natal R, Santos C, Paço J, Analysis of eardrum pathologies using the finite element method, J Mech Med Biol 3:14, 2014.

14. Gentil F, Garbe C, Parente M, Martins P, Santos C, Almeida E, Jorge RN, The biomechanical effects of stapes replacement by prosthesis on the tympano-ossicular chain, Int J Numer Method Biomed Eng 30:1409-1420, 2014.

15. Gentil F, Parente M, Martins P, Garbe C, Paço J, Ferreira A, Tavares J, Jorge RN, The influence of muscles activation on the dynamical behavior of the tympano-ossicular system of the middle ear, Comp Method Biomech Biomed Eng 16:392-402, 2013.

16. Yeoh $\mathrm{OH}$, Characterization of elastic properties of carbon-black-filled rubber vulcanizates, Rubber Chem Technol 63:792-805, 1990.

17. Wen YH, Hsu LP, Chen PR, Lee CF, Design optimization of cartilage myringoplasty using finite element analysis, Tzu Chi Med J 18:370-377, 2006.

18. Schmidt J, Bergander H, Eng L, Experimental and theoretical analysis of shear-force interaction in the non-contact regime with $100 \mathrm{pN}$ force resolution, Appl Surf Sci 157:295-301, 2000. 